\title{
Sarm1 deficiency impairs synaptic function and leads to behavioral deficits, which can be ameliorated by an mGluR allosteric modulator
}

\author{
Chia-Wen Lin ${ }^{1}$, Chiung-Ya Chen ${ }^{1}$, Sin-Jhong Cheng ${ }^{2}$, Hsiao-Tang Hu, ${ }^{1,3}$ and Yi-Ping Hsueh ${ }^{1,3}$ * \\ 1 Institute of Molecular Biology, Academia Sinica, Taipei, Taiwan \\ ${ }^{2}$ Neuroscience Program in Academia Sinica, Taipei, Taiwan \\ ${ }^{3}$ Graduate Institute of Life Sciences, National Defense Medical Center, Taipei, Taiwan
}

Edited by:

Rena Li, Roskamp Institute, USA

Reviewed by:

Jiong Shi, Barrow Neurological

Institute, USA

Francesca Prestori, University of

Pavia, Italy

\section{${ }^{*}$ Correspondence:}

Yi-Ping Hsueh, Institute of Molecular

Biology, Academia Sinica, 128

Academia Road, Section 2, Nankang,

Taipei 115, Taiwan

e-mail: yph@gate.sinica.edu.tw
Innate immune responses have been shown to influence brain development and function. Dysregulation of innate immunity is significantly associated with psychiatric disorders such as autism spectrum disorders and schizophrenia, which are well-known neurodevelopmental disorders. Recent studies have revealed that critical players of the innate immune response are expressed in neuronal tissues and regulate neuronal function and activity. For example, Sarm1, a negative regulator that acts downstream of Tolllike receptor (TLR) 3 and 4, is predominantly expressed in neurons. We have previously shown that Sarm1 regulates neuronal morphogenesis and the expression of inflammatory cytokines in the brain, which then affects learning ability, cognitive flexibility, and social interaction. Because impaired neuronal morphogenesis and dysregulation of cytokine expression may disrupt neuronal activity, we investigated whether Sarm1 knockdown affects the synaptic responses of neurons. We here show that reduced Sarm1 expression impairs metabotropic glutamate receptor (mGluR)-dependent long-term depression (LTD) formation but enhances $\mathrm{N}$-methyl-D-aspartate receptor (NMDAR)-dependent long-term potentiation production in hippocampal CA1 neurons. The expression levels of post-synaptic proteins, including NR2a, NR1, Shank1 and Shank3, are also altered in Sarm1 knockdown mice, suggesting a role for Sarm 1 in the maintenance of synaptic homeostasis. The addition of a positive allosteric modulator of mGluR5, CDPPB, ameliorates the LTD defects in slice recording and the behavioral deficits in social interaction and associative memory. These results suggest an important role for mGluR5 signaling in the function of Sarm1. In conclusion, our study demonstrates a role for Sarm 1 in the regulation of synaptic plasticity. Through these mechanisms, Sarm 1 knockdown results in the impairment of associative memory and social interactions in mice.

Keywords: autism, CDPPB, innate immunity, long-term potentiation, long-term depression, metabotrophic glutamate receptor, $N$-methyl-D-aspartate receptor

\section{INTRODUCTION}

Sarm1 is an evolutionarily conserved adaptor protein that contains, from the $\mathrm{N}$ - to C-terminal region, HEAT/Armadillo motifs, sterile alpha motifs (SAMs) and a Toll/interleukin-1 receptor (Tir) domain. The Tir domain provides Sarml with unique function as a negative regulator of the Toll-like receptor (TLR) 3 and 4 signaling pathways through interaction with TIR-domaincontaining adapter-inducing interferon- $\beta$ (TRIF; Carty et al., 2006). Although Sarm 1 was originally identified in peripheral immune responses (Carty et al., 2006), Sarm1 is predominantly expressed in the brain by neurons (Kim et al., 2007; Chen et al., 2011; Lin et al., 2014). Sarm1 modulates TNF- $\alpha$ production in the brainstem to restrict viral infection (Szretter et al., 2009). Even in the absence of an immune challenge, knockdown of Sarm1 in mice can alter the expression of inflammatory and antiviral cytokines in the brain (Lin et al., 2014), suggesting a neuron-autonomous effect of Sarm1 on cytokine expression. In addition to innate immunity, our previous studies have revealed that Sarm1 participates in neuronal morphogenesis. Through the ASK1-MKK-JNK pathway, Sarm1 can influence microtubule stability and regulate neuronal polarity, axonal outgrowth and dendritic arborization (Chen etal., 2011). Analysis of transgenic Sarm1 knockdown mice also demonstrates that Sarm1 deficiency reduces dendritic arborization and brain size in vivo (Chen et al., 2011).

Aberrant immune responses in the brain and impaired neuronal morphogenesis are both involved in the pathogenesis of neuropsychiatric diseases. Our previous study showed that Sarm1 regulates neuronal morphogenesis (Chen et al., 2011). In addition, IL- $1 ß$ and TNF- $\alpha$, the inflammatory cytokines regulated by Sarm 1 (Lin et al., 2014), have been found to regulate synaptic plasticity (Del Rey et al., 2013; Gruber-Schoffnegger et al., 2013). Taking consideration with several other lines of evidence, Sarm1 is likely associated with neurodevelopmental disorders. First, the human Sarm1 gene is located at chromosome 17q11 (17:26,698,987$26,728,065)$, which is within the autism susceptibility locus 
6 (Auts6, OMIM\%609378, 17:24,000,000-31,800,000). Second, reduced Sarm1 protein levels in the mid-frontal cortex were reported in patients with autism (Azhagiri et al., 2009; Pardo et al., 2009). Finally, the behavioral analysis of Sarm 1 knockdown mice also shows intellectual disability, impaired social behaviors and cognitive inflexibility (Lin and Hsueh, 2014). These behavioral defects resemble the characteristics of patients with autism.

In this study, we further investigate the detailed mechanism associated with the behavioral deficits in transgenic Sarm 1 knockdown mice. A series of electrophysiology studies were thus performed to analyze Sarm1 knockdown mice. We found that metabotropic glutamate receptor (mGluR)-dependent long-term depression (LTD) is impaired and that $N$-methyl-D-aspartate receptor (NMDAR)-dependent long-term potentiation (LTP) is enhanced in Sarm1 knockdown mice. Moreover, pharmaceutical treatments that aimed to correct the synaptic plasticity were able to improve the animals' behavioral defects. Our study suggests that Sarm1 regulates electrophysiological responses through the mGluR pathway, which may be relevant to the function of Sarm1 in cognition.

\section{MATERIALS AND METHODS CALCIUM IMAGING}

Hippocampal neurons were cultured on a coverslip at a density of $2 \times 10^{5}$, and they were transfected with a Sarm 1 shRNA construct (Sarm1i) or a pSuper.neo+GFP vector control (Chen et al., 2011) at six days in vitro (DIV) using calcium phosphate precipitation (Chen and Hsueh, 2012). On DIV9, the neurons were washed with $1 \mathrm{ml}$ of $1 \mathrm{x}$ Hank's balanced salt solution (HBSS; 14025-092, Invitrogen) and then incubated with $2 \mu \mathrm{M}$ Fura-2, AM (F-14185, Invitrogen) in HBSS for $40 \mathrm{~min}$ at $37^{\circ} \mathrm{C}$. Before recording calcium imaging, the cells were washed three times with HBSS to remove excess Fura-2, and then, the cells were transferred to the recording chamber. The images were recorded using an Inverted Fluorescent microscope (Axiovert 200, Carl Zeiss) equipped with a $20 \times /$ NA:0.75/WD $0.6 \mathrm{~mm}$ objective lens and MetaFlour Analyst softwares for acquisition and analysis. The excitation wavelengths of Fura-2 and Fura2- $\mathrm{Ca}^{2+}$ complex are $380 \mathrm{~nm}$ and $340 \mathrm{~nm}$, respectively. The $\mathrm{Ca}^{2+}$ signal in soma was monitored. The images were taken every $2 \mathrm{~s}$ to measure the emission signals. The $340 / 380 \mathrm{~nm}$ excitation ratio was calculated for the indicated intracellular $\mathrm{Ca}^{2+}$ concentration. After recording a baseline, $50 \mu \mathrm{M}$ of glutamate in HBSS was continuously perfused into the recording chamber to trigger excitatory responses. The entire $\mathrm{Ca}^{2+}$ imaging was separated into the baseline, rising and steady phases. The 340/380 ratio in the last 20 cycles $(40 \mathrm{~s})$ was also subtracted from the baseline $340 / 380$ ratio to indicate the synaptic activation upon glutamate treatment.

\section{GOLGI STAINING AND MORPHOMETRY OF DENDRITIC SPINES}

The details for the Golgi staining have been described previously (Chen etal., 2011; Wang etal., 2011). Golgi impregnated hippocampal CA1 neurons were captured using a Zeiss AxioImagerZ1 microscope equipped with an AxioCam HR system (Carl Zeiss), a $63 \times / 1.4$ oil (Plan-APO, Carl Zeiss) objective and AxioVision software (Carl Zeiss). All of the Z-stack images from each neuron were projected into a single panel by Auto-Montage Pro software (Synoptics Ltd). The number of spines in a $20 \mu \mathrm{m}$ segment from three secondary apical dendrites of each CA1 neuron were counted using ImageJ.

\section{PREPARATION OF HIPPOCAMPAL SLICES FOR ELECTROPHYSIOLOGICAL RECORDING}

The recording was performed as described previously (Cheng et al., 2011; Hsu etal., 2011), with some modifications. Male mice that were 8-12 weeks old and postnatal day 25-35 were used for the theta-burst stimulation (TBS)-LTP and (S)-3,5dihydroxyphenylglycine (DHPG)-induced LTD studies, respectively. The mice were decapitated, and their brains were isolated by removing the skull with bone cutting forceps. The brains were then immediately placed in ice-cold artificial cerebrospinal fluid (ACSF) for $3 \mathrm{~min}$ to lower the temperature before dissecting out the hippocampus. ACSF is made with the following (in $\mathrm{mM}$ ): $119 \mathrm{NaCl}, 2.5 \mathrm{KCl}, 1.3 \mathrm{MgSO}_{4}, 26.2 \mathrm{NaHCO}_{3}, 1 \mathrm{NaH}_{2} \mathrm{PO}_{4}$, $2.5 \mathrm{CaCl}_{2}$, and 11 glucose, $\mathrm{pH}$ adjusted to 7.4 and gassing with $95 \% \mathrm{O}_{2} / 5 \%$ CO. Transverse hippocampal slices (450 $\mu \mathrm{m}$ thick) were made by vibrotome (Microslicer ${ }^{\mathrm{TM}}$ DTK-1000, DKS). The slices were kept in an interface-type holding chamber with oxygenated ACSF (95\% $\mathrm{O}_{2} / 5 \% \quad \mathrm{CO}_{2}$ ) at room temperature $\left(24-25^{\circ} \mathrm{C}\right)$ and allowed to recover for at least $90 \mathrm{~min}$ before recording.

\section{EXTRACELLULAR FIELD POTENTIATION RECORDING}

Brain slices were transferred to an immersion-type recording chamber, perfused at $2 \mathrm{ml} / \mathrm{min}$ with ACSF containing $100 \mu \mathrm{M}$ picrotoxin at room temperature. An incision was made between the CA1 and CA3 areas to remove afferent input from CA3. For the extracellular field potential recording, a glass pipette filled with $3 \mathrm{M} \mathrm{NaCl}$ was positioned in the CA1 stratum radiatum area to record the field excitatory post-synaptic potential (fEPSP). Bipolar stainless steel stimulating electrodes (FHC, USA) were placed in the striatum radiatum to stimulate the Schaffer collateral. Stable baseline fEPSP activity was recorded for at least $10 \mathrm{~min}$ by applying a short-duration current stimulation pulse $(\sim 40 \mu \mathrm{s})$ at a predetermined intensity every $15 \mathrm{~s}$. A TBS protocol, with four pulses at $100 \mathrm{~Hz}$ in a burst and 10 bursts in a train, was delivered once for LTP induction. A paired-pulse stimulation $(50 \mathrm{~ms}$ intervals) at $1 \mathrm{~Hz}$ for $20 \mathrm{~min}$ (1200 trains) with D, L-AP5 applied during recording was used to measure mGluR5-dependent LTD. For paired-pulse facilitation, the stimulation intervals were in a sequence of 50,100,200, 300, 500 and $30 \mathrm{~ms}$, with an average of 20 traces. All of the signals were filtered at $1 \mathrm{kHz}$ using the low-pass Bessel filter that was provided with the amplifier, and the signals were digitized at $10 \mathrm{kHz}$ using a CED micro 1401 interface running Signal software (Cambridge Electronic Design, Cambridge, UK). The initial slopes of the fEPSP were measured for data analysis. The synaptic responses were normalized to the average of the baseline. The slope of the fEPSPs recorded during the last 10 min after a different pulse stimulation was averaged for statistical comparisons. All data are presented as means \pm SEMs. Statistical significance was tested by unpaired $t$-test. $P<0.05$ was considered statistically significant. 


\section{WHOLE-CELL RECORDING FOR THE NMDA/AMPA RATIO}

Adult male hippocampal slices were cut perpendicularly to the long axis in thickness of $300 \mu \mathrm{m}$ for the whole-cell voltage-clamp recordings. The slices were transferred to an immersion-type recording chamber mounted on an upright microscope (BX50WI, Olympus Optical Co., Ltd, Tokyo, Japan) equipped with 40x water-immersion objectives, a Nomarski optic system, an infrareddifferential interference-contrast microscopy and a CCD camera (Sony XC-EI50, Japan). The oxygenated ACSF was continuously perfused at $1-2 \mathrm{ml} / \mathrm{min}$. Recorded CA1 pyramidal neurons were selected based on their morphology and location. The patch pipettes pulled from borosilicate glass tubing $(1.5 \mathrm{~mm}$ outer diameter, $0.86 \mathrm{~mm}$ inner diameter; G150F-4, Warner Instruments) had a resistance of 5-8 $\mathrm{M} \Omega$ when filled with internal solution which consisting of the following (in $\mathrm{mM}$ ): $131 \mathrm{~K}$-gluconate, 20 $\mathrm{KCl}, 10$ HEPES, 2 EGTA, $8 \mathrm{NaCl}, 2$ ATP, $0.3 \mathrm{GTP}$, and 6.7 biocytin, with the $\mathrm{pH}$ adjusted to 7.2 by $\mathrm{KOH}$ and the osmolarity to 300-305 mOsm. Recordings were made at room temperature $\left(24-25^{\circ} \mathrm{C}\right)$ with a patch amplifier (Multiclamp $700 \mathrm{~B}$; Axon Instruments, Union City, CA, USA). For voltage-clamp recordings, the series resistance (Rs) was continuously monitored by applying a voltage pulse of $1 \mathrm{mV}$ and was not compensated for. Data were discarded when the Rs varied by $>20 \%$ from its original value during the recording. The membrane capacitance of recorded neurons was $30.44 \pm 3.22 \mathrm{pF}$ for WT neurons and $36.06 \pm 6.96 \mathrm{pF}$ for Sarm1 knockdown neurons $(n=6)$. The membrane resistance of recorded neurons was $327.89 \pm 41.70 \mathrm{M} \Omega$ for WT neurons and $353.10 \pm 54.44 \mathrm{M} \Omega$ for Sarm1 knockdown neurons $(n=6)$. All signals were low-pass filtered at a corner frequency of $1 \mathrm{kHz}$ and digitized at $10 \mathrm{kHz}$ using a Micro 1401 interface (Cambridge Electronic Design). Data were collected using Signal software (Cambridge Electronic Design, Cambridge, UK).

For the NMDAR/AMPAR ratio experiments, AMPARmediated and NMDAR-mediated excitatory post-synaptic currents (EPSCs) were analyzed in two steps for each neuron. First, the stable synaptic responses (EPSCs) were obtained by holding a neuron at $-70 \mathrm{mV}$. The amplitude of these responses was primarily generated by the $\alpha$-Amino-3-hydroxy-5-methyl4-isoxazolepropionic acid (AMPA) receptor because the bathapplied $10 \mu \mathrm{M}$ 6-cyano-7-nitroquinoxaline-2,3-dione (CNQX) in ACSF completely blocked the current. Next, the holding potential was changed to $+40 \mathrm{mV}$ to measure the synaptic responses mediated by the NMDA receptors, which were later blocked by $50 \mu \mathrm{M}$ AP5 with CNQX perfusion. Twenty traces at each holding potential were averaged to analyze the current mediated by each specific receptor. For the NMDA/AMPA ratio, AMPA or NMDA EPSCs were measured by the amplitude of the peak current.

\section{CHEMICALS AND TREATMENT IN THE ELECTROPHYSIOLOGY STUDY}

The chemicals used for the ACSF and internal solutions were purchased from Merck and Sigma. DL-APV, CNQX, picrotoxin, DHPG, and CDPPB [3-cyano- $N$-(1,3-diphenyl-1H-pyrazol-5-yl) benzamide] were purchased from Tocris. All of the drugs were prepared in a high-concentration stock that was kept in an $-80^{\circ} \mathrm{C}$ freezer. The chemicals were added to an ACSF bath prior to being applied.

\section{IMMUNOBLOTTING ANALYSIS}

The entire hippocampus was homogenized with a pellet pestle in lysis buffer containing $50 \mathrm{mM}$ Tris (pH 7.4), $320 \mathrm{mM}$ sucrose, $2 \mathrm{mM}$ dithiothreitol, $2 \mu \mathrm{g} / \mathrm{ml}$ leupeptin, $2 \mu \mathrm{g} / \mathrm{ml}$ leupeptin, $2 \mu \mathrm{g} / \mathrm{ml}$ pepstatin-A, $2 \mu \mathrm{g} / \mathrm{ml}$ aprotinin, $1 \mathrm{mM}$ buffer tosylphenylalanylchloromethane, and $2 \mathrm{mM}$ phenylmethylsulfonyl fluoride and was then centrifuged at $800 \mathrm{x} g$ for $10 \mathrm{~min}$ at $4^{\circ} \mathrm{C}$. The supernatant was boiled at $55^{\circ} \mathrm{C}$ in SDS sample buffer and separated by $7.5 \%$ or $10 \%$ SDSPAGE. After transfer, the membrane was treated with a blocking solution $(2.5 \%$ non-fat milk in $0.3 \%$ TBST $)$ followed by incubation with primary antibodies at $4^{\circ} \mathrm{C}$ overnight. The proteins were detected with HRP-conjugated goat secondary antibody and developed with Western Lightning Plus ECL (PerkinElmer). The blot images were developed using ImageQuant ${ }^{T M}$ LAS 4000 (GE Health care Life Sciences, USA) and X-ray film (Fujifim) without modification of contrast or brightness.

\section{BEHAVIOR ANALYSIS}

All of the animal experiments were carried out with the approval of the Academia Sinica Institutional Animal Care and Utilization Committee. Sarm1 knockdown mice were generated in a C57BL/6J background (Chen etal., 2011). The animals used in the behavioral assays were the offspring of transgenic males and wild-type C57BL/6J females to eliminate any maternal effects of Sarm1 knockdown on offspring behavior. Male littermate mice were used in the behavioral assays to avoid variations due to the estrus cycle. All of the procedures for the behavioral analyses have been described previously (Chung etal., 2011; Lin and Hsueh, 2014), with minor modifications. The behavioral testing was performed at 8-16 weeks of age. All of the animals were housed in mixed-genotype groups of 3-5 mice per cage. The animals were acclimatized to the test room for at least 1 week prior to the behavioral assays. A $12 \mathrm{~h}$ light/dark cycle (lights off at 20:00) was maintained in the test room. Food and water were accessed ad libitum. For the reciprocal social interaction assays, each experimental mouse was independently housed for 1 week before the test. The tests were performed between 14:00 and 19:00, prior to the onset of the dark phase. For the rescue experiments, CDPPB was dissolved in DMSO at a concentration of $14.6 \mathrm{mg} / \mathrm{ml}(40 \mathrm{mM})$, and it was further diluted in polyethylene glycol-400 (PEG400, Sigma) at a ratio of 1:8 to increase its solubility. Wild-type or Sarm1 knockdown mice received an intraperitoneal (i.p.) injection of CDPPB at a dose of $10 \mathrm{mg} / \mathrm{kg}$, or they received the same volume of a DMSO-PEG400 mixture as a vehicle control. The i.p. injections were performed six hours before the day 0 training for contextual fear conditioning or reciprocal social interaction to give sufficient recovery time from the illness caused by viscous PEG.

\section{STATISTICAL ANALYSES}

Unpaired $t$-tests were performed to analyze the differences between WT and transgenic ( $\mathrm{Tg}$ ) animals using GraphPad Prism 5.0 (GraphPad Software, La Jolla, CA, USA). Two-way ANOVA was used to analyze Figure 6. For all comparisons, a $P$ value $<0.05$ was considered significant. 


\section{RESULTS}

\section{Sarm1 KNOCKDOWN LEADS TO HYPERSYNAPTIC RESPONSES UPON} GLUTAMATE STIMULATION IN HIPPOCAMPAL NEURONS

To investigate whether Sarm1 knockdown influences synaptic function, $\mathrm{Ca}^{2+}$ imaging using cultured hippocampal neurons was performed to monitor neuronal activity induced by glutamate treatment. The neurons were transfected with a Sarm 1 knockdown construct, Sarm1i (Chen et al., 2011), at DIV 6 and then subjected to $\mathrm{Ca}^{2+}$ image recording at DIV 9. The Sarm1 knockdown construct co-expresses green fluorescence protein (GFP). Thus, the GFP signal provides a marker for neuronal Sarm1 knockdown and outlines neuronal morphology. We first confirmed that similar to previous findings (Chen et al., 2011), knockdown of Sarm1 impaired dendritic arborization (Figure 1A). The $\mathrm{Ca}^{2+}$ influx induced by glutamate stimulation was investigated in neurons that were transfected with a vector control or Sarm1i at DIV 9 by monitoring intracellular $\mathrm{Ca}^{2+}$ concentrations, as indicated by Fura- 2 . After recording the baseline of intracellular calcium concentration, glutamate was added into the culture to induce calcium influx through NMDAR (Figure 1B). We found that compared the calcium concentration before and after glutamate stimulation, Sarm1 knockdown obviously induced more calcium influx after glutamate treatment (Figure 1C). The entire response was also divided into three phases including baseline, rising and steady phases (Figure 1B). For the baseline and rising phases, calcium influx was comparable between control and Sarm1 knockdown neurons (Figure 1D). For the steady phase, the calcium concentration of Sarm1 knockdown neurons was noticeably higher than that of control neurons. To make sure that the activation of NMDAR does not induce cytotoxicity during recording, we also monitored whether the calcium concentration is able to return to the baseline after NMDAR activation and further respond to calcium ionophore A23187 (Figure 1E). Indeed, the cultures were still healthy after induction of NMDAR opening and were able to return to the baseline and further respond to calcium ionophore A23187 (Figure 1E). Taken together, the results of calcium imaging

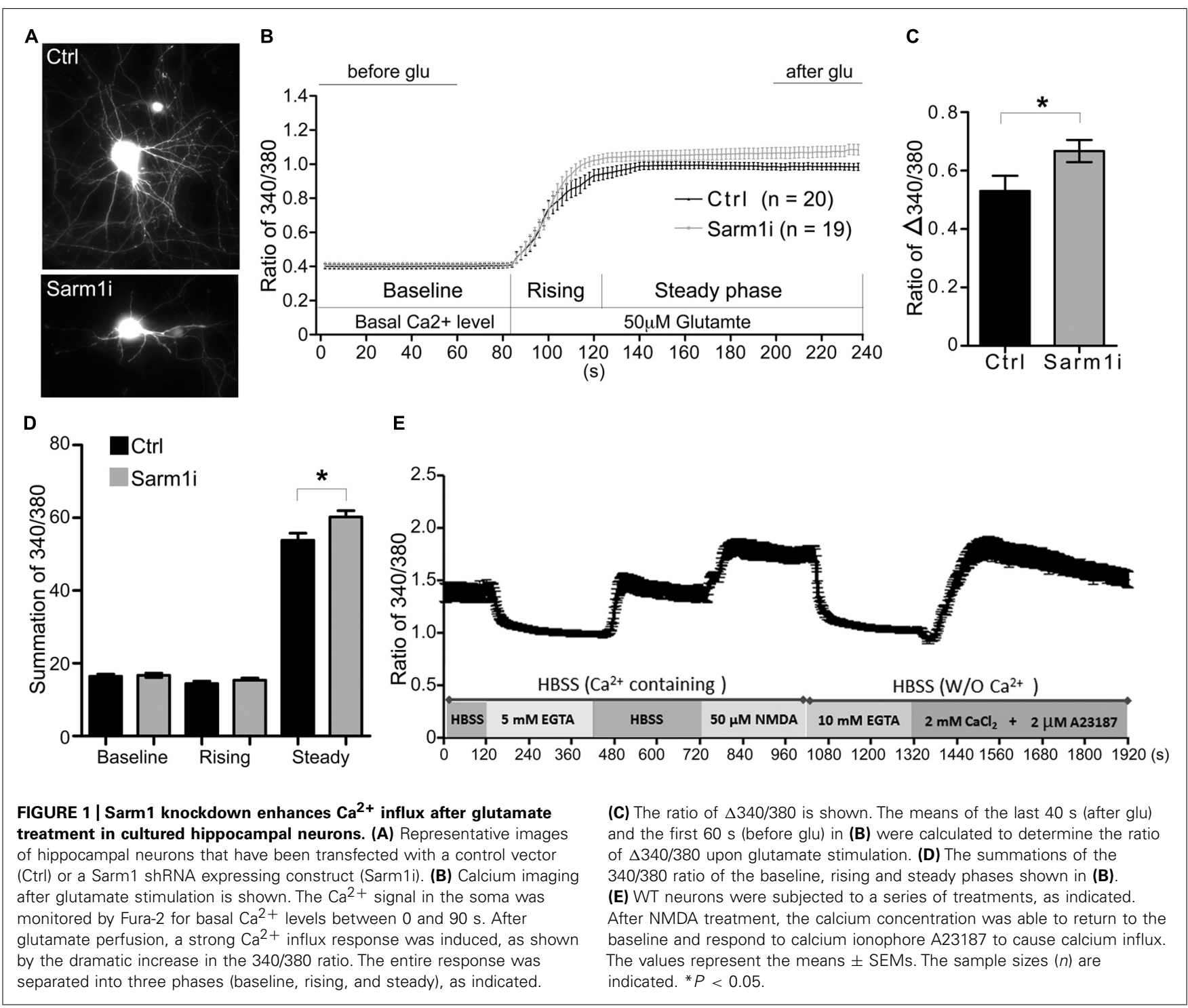


indicated that neurons with Sarm1 knockdown had a higher $\mathrm{Ca}^{2+}$ influx after glutamate stimulation compared to the vectortransfected control. The enhanced responses to neuronal stimulation suggested that Sarm1 knockdown can change the synaptic composition or the downstream signaling pathway following stimulation.

\section{Sarm1 KNOCKDOWN INCREASES THE SPINE DENSITY OF CA1 NEURONS AND ENHANCES NMDAR-DEPENDENT SYNAPTIC PLASTICITY IN Sarm1 KNOCKDOWN MICE}

Because spine morphology is linked with and changed by synaptic activity, we then examined whether Sarm1 deficiency alters the morphology and density of dendritic spines of the CA1 neurons. Golgi stain was used to analyze Sarm1 knockdown mice, which were established previously (Chen et al., 2011). The results showed that the dendritic spines of Sarm1 knockdown neurons tended to be elongated in their morphology (Figure 2A). However, the length and width of individual dendritic spines were immeasurable because the spines were bunched together. Thus, we only quantified the density of the dendritic spines. The data showed that Sarm 1 knockdown resulted in a higher density of dendritic spines in CA1 neurons (Figures 2B,C). This increase in dendritic spine density echoes the increase of calcium concentration in Sarm1 knockdown neurons after glutamate stimulation.

We then conducted a series of electrophysiology studies to corroborate the involvement of Sarm 1 in synaptic plasticity. An extracellular field recording in Schaffer collateral-CA1 synapses was performed using acute hippocampal slices. We first examined the NMDAR-dependent LTP induced by TBS. Consistent with the higher calcium influx after glutamate stimulation and the higher dendritic spine density, the fEPSPs of Sarm1 knockdown hippocampal slices were enhanced by approximately $25 \%$ compared

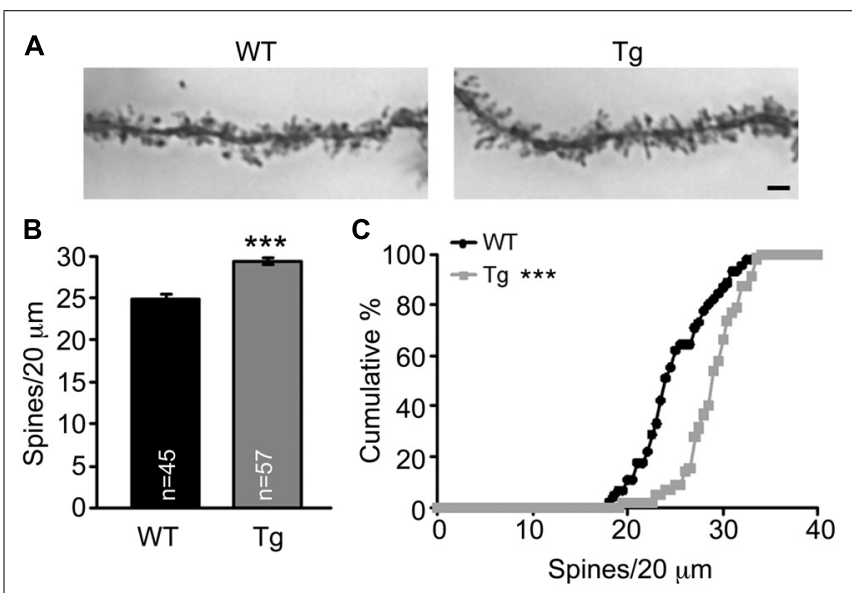

FIGURE 2 | Sarm 1 knockdown increases dendrite spine density in the hippocampal CA1 region. (A) The representative images of CA1 neuronal Golgi stains are shown. (B) The mean ( \pm SEM) and (C) cumulative probability of the numbers of dendritic spines were compared between WT and Sarm 1 knockdown CA1 neurons. The scale bars are set at $5 \mu \mathrm{m}$. The sample sizes $(n)$ of the dendrite number are indicated in the panels. The data were collected from 15 to 19 neurons from 4 WT to 4 Sarm1 knockdown mice, respectively. ${ }^{* *} P<0.001$. to the wild-type littermates (Figure 3A). It suggests that Sarm1 knockdown results in a hyper-LTP.

To dissect the origin of the disturbed NMDAR-dependent LTP, paired-pulse stimulation was used to test the ability of presynaptic terminals to release vesicles. The ratio of paired-pulse stimulation indicated that vesicle release was comparable between Sarm1 knockdown and wild type neurons (Figure 3B). It suggests that Sarm 1 knockdown hippocampi have normal presynaptic function. We then analyzed the relative contribution of the NMDA and AMPA receptors to the EPSCs that were evoked by stimulation from the Schaffer collateral afferent. We found that the NMDA/AMPA ratio was significantly increased in Sarm1 knockdown neurons (Figure 3C). Taken together, these data indicate that reduced Sarm1 expression affects the synaptic response through a post-synaptic mechanism, which regulates the conductivity of NMDAR and AMPAR.

We also analyzed the composition of synaptic proteins by isolating crude synaptosomal fractions (P2) from adult wild type and Sarm1 knockdown hippocampi. The results showed that the expression levels of presynaptic proteins, including synapsin, synaptotagmin, SVP38, NSF and Munc18, were not altered (Figure 4A), echoing the normal paired-pulse facilitation in Sarm 1 knockdown brains. The only significant differences were the expression of post-synaptic scaffold proteins and NMDA receptor subunits, including Shank1, Shank3, NR1, and NR2a (Figures 4B,C). The increases were small $(\sim 12-25 \%)$ but significant. These changes of post-synaptic protein levels provide a potential molecular explanation for hyper-NMDAR-dependent LTP in Sarm1 knockdown mice.

\section{Sarm1 KNOCKDOWN ALSO RESULTS IN IMPAIRED mGIuR-DEPENDENT LTD}

In addition to NMDAR-dependent LTP, we investigated LTD in the Sarm1 knockdown hippocampus. We were interested in the mGluR-dependent LTD because its involvement in synaptic pathophysiology has been confirm in two syndromic forms of autism with intellectual disability, tuberous sclerosis complex $\left(\mathrm{TSC}^{+/-}, \mathrm{TSC}^{+/-}\right)$and fragile X syndrome $\left(\mathrm{Fmrl}^{-/-}\right.$; Bear et al., 2004; Zoghbi and Bear, 2012). Activation of mGluR triggers rapid local translation of proteins required for the endocytosis of AMPA receptors. This activity reduces the surface expression of AMPA receptors and leads to the formation of mGluR-induced LTD. Because Sarm1 has been shown to influence the ASK1MKK-JNK/p38 pathway in neurons (Chuang and Bargmann, 2005; Chen et al., 2011), which is required for mGluR downstream signaling (Li et al., 2007; Moult et al., 2008), we asked whether Sarm1 could also participate in the synaptic activity that was regulated by mGluR. Two independent protocols were used to measure mGluR-LTD, including chemically and electrically induced LTD, either by treating with DHPG, an agonist of group I mGluR, or by paired pulse-low frequency stimulation (PP-LFS). The results of both experiments indicated that none of stimulations was able to induce LTD in Sarm1 knockdown brains, because compared to a wild-type control, Sarm1 knockdown brains exhibited a greatly reduced magnitude of DHPGand PP-LFS-induced LTD in the hippocampus (Figures 5A,B). To confirm that the deficiency of mGluR signaling in Sarm1 

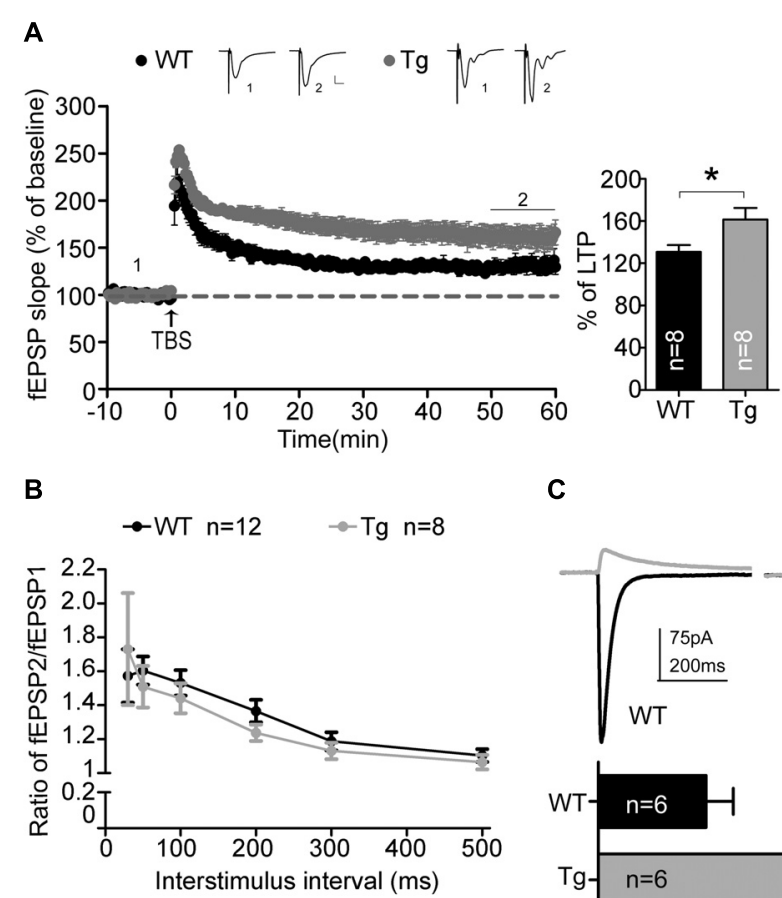

C

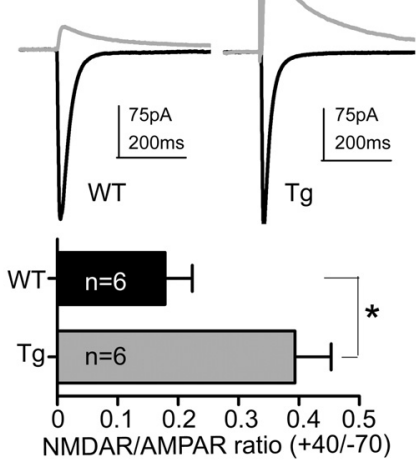

FIGURE 3 | Sarm 1 knockdown enhances NMDAR-dependent LTP in hippocampi. (A) Theta burst stimulation-induced LTP was recorded in the hippocampal CA1 region. The fEPSPs were recording in the CA1 and plotted against time. The representative traces shown at the top are an average of 40 fEPSPs from the baseline (1) and the last 10 min of recording (2). Each trace is the average of 40 fEPSPs from the baseline. The last $10 \mathrm{~min}$ of recording are further subjected for quantitative analysis. The horizontal dashed lines indicate the average value of the normalized amplitude during the baseline period. Calibration: $0.2 \mathrm{mV}, 10 \mathrm{msec}$. The histogram summarizing the average fEPSP of the last $10 \mathrm{~min}$ of recording is shown in the right panel. The data represent the mean plus SEM (for histograms) or \pm SEM (for fEPSP slope). ${ }^{*} P<0.05$. (B) The paired-pulse facilitation was similar between Sarm1 knockdown and WT mice. The data represent the mean \pm SEM. (C) The representative NMDAR (indicated by the gray line) and AMPAR (indicated by the black line) mediated currents from WT and Sarm 1 knockdown CA1 neurons are shown in the upper panel. The ratio of NMDA to AMPA current is shown in the lower panel. The data indicate the mean plus SEM. ${ }^{*} P<0.05$. The sample sizes $(n)$ are indicated. knockdown mice, CDPPB, was added prior to the administration of DHPG. CDPPB functions as a positive allosteric modulator of mGluR5, which facilitates the effect of DHPG on mGluR. Thus, if the defect of Sarm1 knockdown neurons in response to DHPG is due to the deficit of mGluR signaling, the treatment of CDPPB is expected to rescue the defects in LTD (Auerbach et al., 2011; Verpelli et al., 2011). Indeed, enhancing mGluR5 signaling by CDPPB reversed the LTD defect in Sarm 1 knockdown (Figure 5C). It confirmed that the deficits in mGluR5 signaling were involved in the LTD impairment of Sarm1 knockdown mice.

\section{CDPPB TREATMENT AMELIORATES THE BEHAVIORAL DEFECTS IN Sarm1 KNOCKDOWN MICE}

A recent study that focused on fragile $\mathrm{X}$ and tuberous sclerosis mouse models suggests that there is shared synaptic pathology in mGluR signaling (Auerbach et al., 2011). In particular, $\mathrm{TSC}^{+/-}$mutant mice display reduced mGluR-LTD responses, which are associated with intellectual disabilities, and these symptoms can be improved by enhancing mGluR signaling (Auerbach et al., 2011). Because Sarm1 knockdown mice are characterized by autistic phenotypes (Lin and Hsueh, 2014) and because the data above showed an impairment of mGluR-dependent LTD in Sarml knockdown mice, we asked whether a pharmacological treatment could reverse the defects in Sarm1 knockdown mice, specifically dysregulated mGluR-dependent LTD and behavioral defects. Reciprocal social interaction and contextual fear conditioning were examined. We have previously demonstrated that Sarm1 knockdown affects these two behavior paradigms in mice (Lin and Hsueh, 2014). CDPPB was injected i.p. into Sarm1 knockdown and WT littermate mice prior to the behavioral assays (Figures 6A,B). Consistent with the effects of CDPPB on electrophysiology (Figure 4C), we found that the systemic administration of CDPPB also ameliorated the behavioral defects observed in Sarm1 knockdown mice during contextual fear conditioning (Figure 6A) and reciprocal social interaction (Figure 6B). These results strengthen the link between mGluR-dependent LTD and Sarm1-regulated neuronal function, and they provide a possible treatment to ameliorate the behavioral defects caused by Sarm 1 deficiency.

\section{DISCUSSION}

In this report, we demonstrate that Sarm1 knockdown causes synaptic dysfunction, including increased $\mathrm{Ca}^{2+}$ influx upon 
A

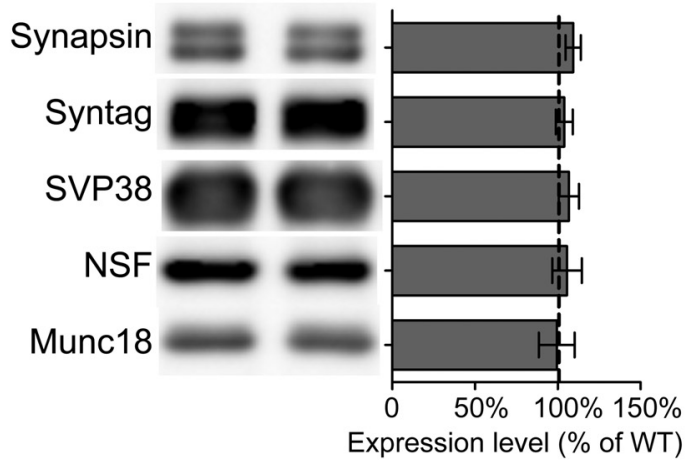

B

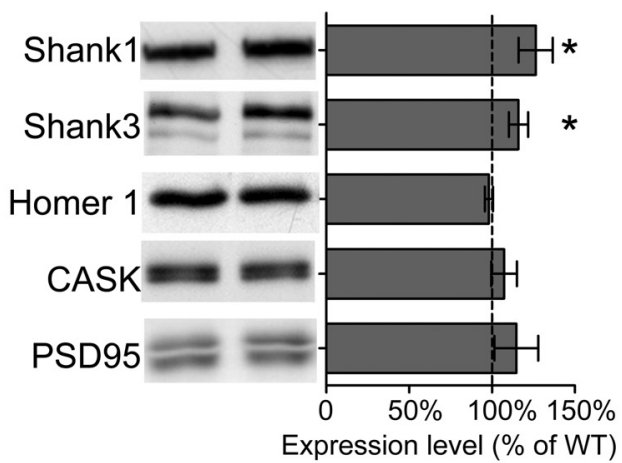

C

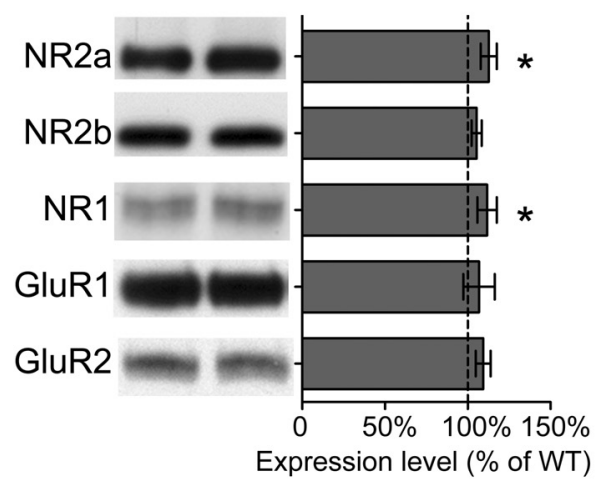

FIGURE 4 | Expression of some synaptic proteins are altered in Sarm1 knockdown mice. (A) Presynaptic proteins. (B) Post-synaptic protein. (C) Glutamate receptors. The synaptosomal fractions of the hippocampi were analyzed by immunoblotting with antibodies, as indicated. Five animals for each genetic background were used for analysis. The data represent the mean \pm SEM normalized to the WT levels. ${ }^{*} P<0.05$.

glutamate treatment in vitro as well as enhanced NMDARdependent LTP and impaired mGluR-dependent LTD in hippocampi. The behavioral rescue by $C D P P B$, a positive allosteric modulator of mGluR, further supports a role for mGluR signaling in the pathogenesis of autistic-like behaviors that are caused by Sarm 1 knockdown. Metobotrophic GluR signaling activity is known to mediate spine shrinkage and degradation (Oh et al., 2013; Ramiro-Cortes and Israely, 2013), which may account for the increased spine density that was observed in the CA1 pyramidal neurons. Our data also suggest that there are changes in postsynaptic protein composition, including NR1, NR2a, and Shank

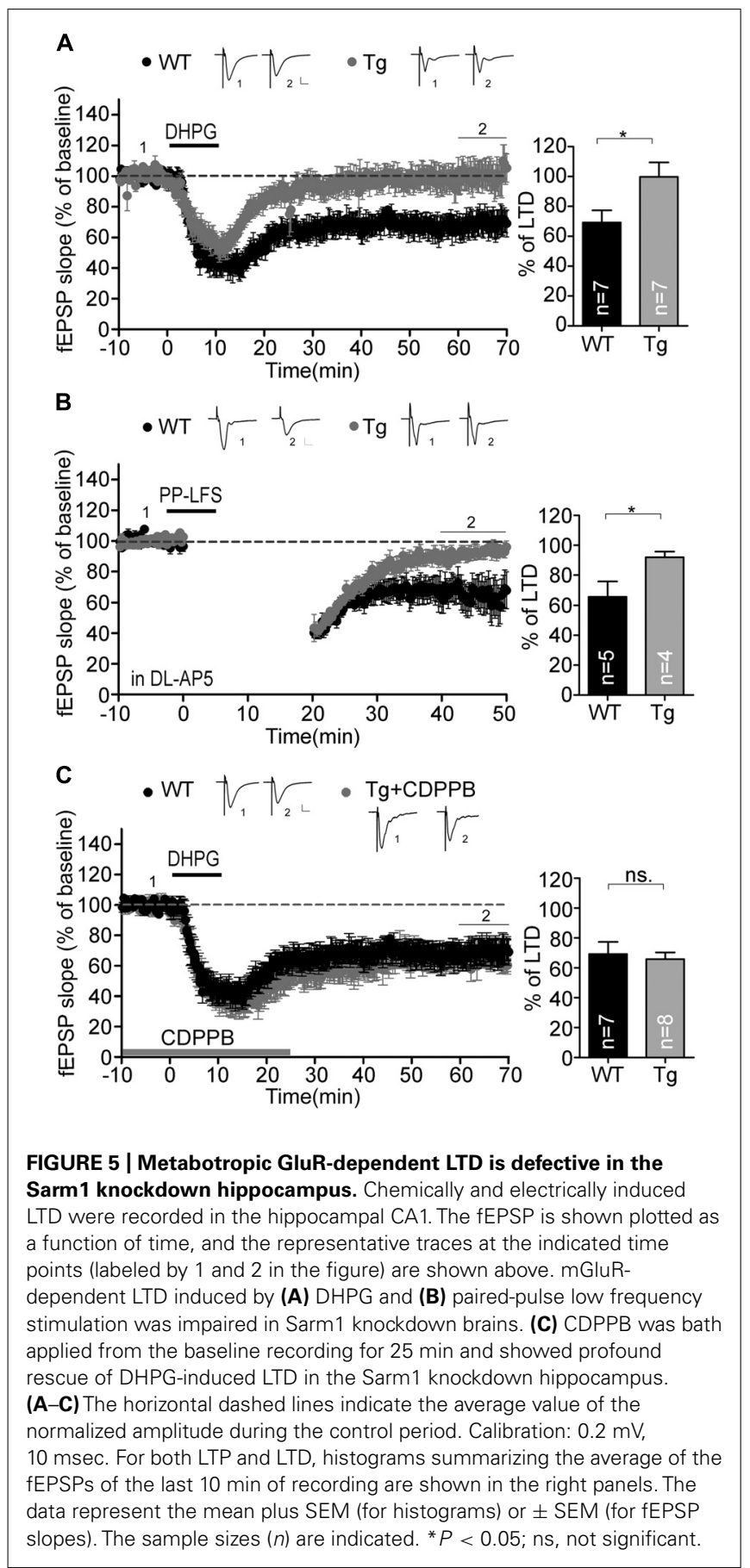

proteins, in Sarm 1 knockdown brains. These synaptic proteins not only directly contribute to synaptic responses but are also known to be involved in the etiology of autism spectrum disorder (ASD). For example, all of the members of the Shank protein family that indirectly link NMDAR and mGluR were identified as autism causative genes (Durand etal., 2007; Hung etal., 2008; Berkel et al., 2010; Pinto et al., 2010; Peca et al., 2011; Sato et al., 2012; Schmeisser et al., 2012; Won et al., 2012). Mutations in the NR2b gene were consistently identified in patients with autism (O'Roak et al., 2012). These observations support the current hypothesis 

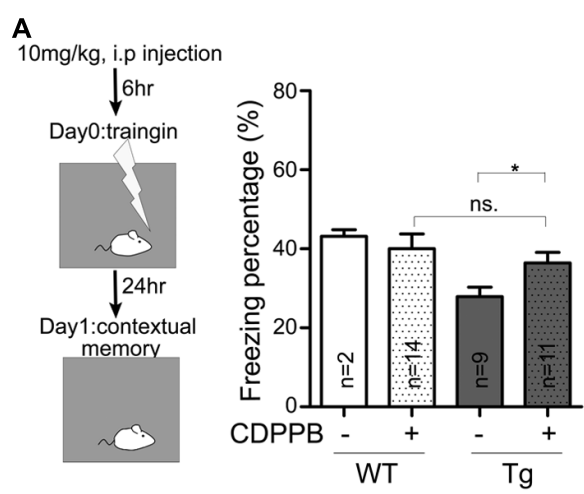

FIGURE 6 | Enhancement of mGluR5 signaling ameliorates the behavioral defects in Sarm1 knockdown mice. CDPPB $(10 \mathrm{mg} / \mathrm{kg}$ of animal body weight) or a vehicle control was intraperitonally injected into mice six hours before the behavioral assay. (A) The results of contextual fear conditioning are
B

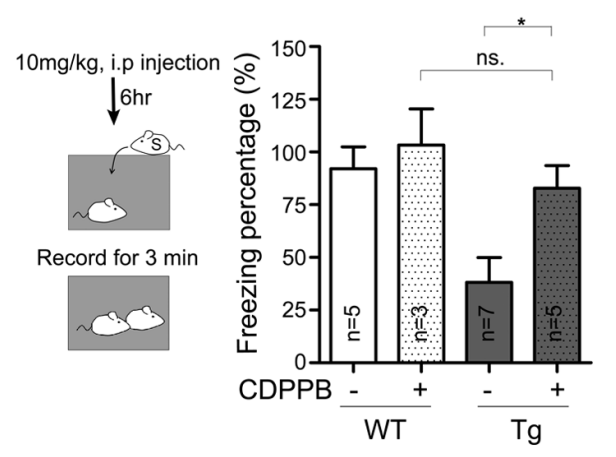

shown. (B) The results of reciprocal social interaction are shown. CDPPB did not obviously influence the behaviors of WT littermates. However, CDPPB rescued the defects observed in Sarm 1 knockdown mice. The sample sizes $(n)$ are indicated in the column. ${ }^{*} P<0.05$; ns, not significant. that the intellectual disabilities associated with autism are caused by unbalanced synaptic activity. Both overly strong or overly weak synaptic function can harm neural plasticity and results in autisticlike behaviors and impaired intellectual performance (Auerbach et al., 2011; Sui and Chen, 2012; Zoghbi and Bear, 2012; Lin et al., 2013; Nuytens et al., 2013). However, the detailed mechanisms underlying the role of Sarm1 in LTD and LTP have yet to be determined. For example, it will be intriguing to further elucidate whether Sarm 1 regulates mGluR and NMDAR signaling through independent mechanisms or primarily through an effect on the mGluR pathway that modulates the NMDAR response indirectly. Because Shank proteins were upregulated in Sarm1 knockdown neurons and because Shanks link NMDAR to mGluR, Sarm1 knockdown may also influence both NMDAR and mGluR via Shanks.

Based on previous studies, Sarm1 likely regulates synaptic plasticity through both direct and indirect mechanisms. Our previous study showed that Sarm 1 is widely distributed in different neuronal subcellular regions, including in the synapses (Chen etal., 2011). Sarm1 puncta were partially co-localized with PSD-95 (Chen et al., 2011). The synaptic localization of Sarm1 can be maintained by direct interaction with syndecan2, a transmembrane heparan sulfate proteoglycan that is highly enriched at the synapse. Synaptic Sarm1 may recruit signaling molecules, such as the components of the MKK4-JNK pathway, to synapses. In C. elegans, the synaptic localization of Tir-1, a Sarm 1 homolog, is determined by the interaction between its SAM domain and UNC-43 (CaMKII homolog; Chuang and Bargmann, 2005). In mammalian neurons, CaMKII is also a key component in synaptic plasticity, and it is concentrated at the post-synaptic region (Lisman et al., 2002; Lee et al., 2009). It seems likely that Sarm1 also interacts directly with CaMKII to control synaptic plasticity.

Sarm1 also regulates neuronal morphogenesis (Chen et al., 2011) and the expression of inflammatory cytokines in the brain (Lin etal., 2014). In addition to dendritic arborization and axonal differentiation, we showed here that Sarm1 knockdown can also influence dendritic spine density. Because proper neuronal morphology is essential for effective communication between neurons, the abnormalities of neuronal morphology caused by Sarm1 knockdown may result in dysregulated circuits between hippocampal CA1 and CA3, thus impairing synaptic plasticity in Sarm1 knockdown mice. On the other hand, Sarm1 knockdown causes dysregulated cytokine expression in the brain, including changes in IL-1 $\beta$, IL-12, CCL5, TNF$\alpha$, and IFN- $\beta$ production (Lin et al., 2014). IL- $1 \beta$ and TNF- $\alpha$ are known to regulate synaptic plasticity (Del Rey et al., 2013; Gruber-Schoffnegger etal., 2013). Thus, it is also likely that inflammatory cytokines mediate Sarm 1 effects on synaptic plasticity.

In the rescue experiments, $\mathrm{CDPPB}$ treatment was able to ameliorate the behavioral defects found in the Sarm1 knockdown mice, suggesting a critical role for mGluR signaling in the Sarm1 pathway. Because of the function of Sarm1 in innate immune responses, our data also reveals a potential treatment for inflammation-related neurological disorders by using mGluR agonists.

\section{AUTHOR CONTRIBUTIONS}

Chia-Wen Lin designed and carried out the experiments, including calcium imaging, immunoblots, electrophysiological recording and behavioral analysis, and drafted the manuscript. ChiungYa Chen designed and carried out the Golgi stain and drafted the manuscript. Sin-Jhong Cheng designed and carried out the electrophysiological recordings and drafted the manuscript. Hsiao-Tang $\mathrm{Hu}$ designed and carried out the calcium imaging experiment shown in Figure 1E. Yi-Ping Hsueh designed the experiments and drafted the manuscript. All of authors read and approved the final version of manuscript.

\section{ACKNOWLEDGMENTS}

This work was supported by grants from Academia Sinica (AS-103TP-B05 to Yi-Ping Hsueh) and the National Science Council (NSC 102-2321-B-001-029 and 102-2321-B-001-054 to Yi-Ping Hsueh). Chiung-Ya Chen was supported by National Science Council (NSC 102-2811-B-001-037). 


\section{REFERENCES}

Auerbach, B. D., Osterweil, E. K., and Bear, M. F. (2011). Mutations causing syndromic autism define an axis of synaptic pathophysiology. Nature 480, 63-68. doi: 10.1038/nature10658

Azhagiri, A., Lawler, C., Zea-Vera, A., and Pardo, C. (2009). “TLR signaling pathways in brain tissue from patients with autism," in Annual Meeting of the Society for Neuroscience, (Washington, DC: The Society for Neuroscience).

Bear, M. F., Huber, K. M., and Warren, S. T. (2004). The mGluR theory of fragile X mental retardation. Trends Neurosci. 27, 370-377. doi: 10.1016/j.tins.2004. 04.009

Berkel, S., Marshall, C. R., Weiss, B., Howe, J., Roeth, R., Moog, U., et al. (2010). Mutations in the SHANK2 synaptic scaffolding gene in autism spectrum disorder and mental retardation. Nat. Genet. 42, 489-491. doi: 10.1038/ng.589

Carty, M., Goodbody, R., Schroder, M., Stack, J., Moynagh, P. N., and Bowie, A. G. (2006). The human adaptor SARM negatively regulates adaptor protein TRIF-dependent Toll-like receptor signaling. Nat. Immunol. 7, 1074-1081. doi: 10.1038/ni1382

Chen, C. Y., Lin, C. W., Chang, C. Y., Jiang, S. T., and Hsueh, Y. P. (2011). Sarm1, a negative regulator of innate immunity, interacts with syndecan-2 and regulates neuronal morphology. J. Cell Biol. 193, 769-784. doi: 10.1083/jcb.2010 08050

Chen, Y. K., and Hsueh, Y. P. (2012). Cortactin-binding protein 2 modulates the mobility of cortactin and regulates dendritic spine formation and maintenance. J. Neurosci. 32, 1043-1055. doi: 10.1523/JNEUROSCI.4405-11.2012

Cheng, S. J., Chen, C. C., Yang, H. W., Chang, Y. T., Bai, S. W., Yen, C. T., et al. (2011). Role of extracellular signal-regulated kinase in synaptic transmission and plasticity of a nociceptive input on capsular central amygdaloid neurons in normal and acid-induced muscle pain mice. J. Neurosci. 31, 2258-2270. doi: 10.1523/JNEUROSCI.5564-10.2011

Chuang, C. F., and Bargmann, C. I. (2005). A Toll-interleukin 1 repeat protein the synapse specifies asymmetric odorant receptor expression via ASK1 MAPKKK signaling. Genes Dev. 19, 270-281. doi: 10.1101/gad.1276505

Chung, W. C., Huang, T. N., and Hsueh, Y. P. (2011). Targeted deletion of CASK-interacting nucleosome assembly protein causes higher locomotor and exploratory activities. Neurosignals 19, 128-141. doi: 10.1159/000327819

Del Rey, A., Balschun, D., Wetzel, W., Randolf, A., and Besedovsky, H. O. (2013). A cytokine network involving brain-borne IL-1beta, IL-1ra, IL-18, IL-6, and TNFalpha operates during long-term potentiation and learning. Brain Behav. Immun. 33, 15-23. doi: 10.1016/j.bbi.2013.05.011

Durand, C. M., Betancur, C., Boeckers, T. M., Bockmann, J., Chaste, P., Fauchereau, F., et al. (2007). Mutations in the gene encoding the synaptic scaffolding protein SHANK3 are associated with autism spectrum disorders. Nat. Genet. 39, 25-27. doi: $10.1038 /$ ng 1933

Gruber-Schoffnegger, D., Drdla-Schutting, R., Honigsperger, C., Wunderbaldinger, G., Gassner, M., and Sandkuhler, J. (2013). Induction of thermal hyperalgesia and synaptic long-term potentiation in the spinal cord lamina I by TNFalpha and IL-1beta is mediated by glial cells. J. Neurosci. 33, 6540-6551. doi: 10.1523/jneurosci.5087-12.2013

Hsu, J. C., Cheng, S. J., Yang, H. W., Wang, H. J., Chiu, T. H., Min, M. Y., et al. (2011). Bidirectional synaptic plasticity induced by conditioned stimulations with different number of pulse at hippocampal CAl synapses: roles of $N$-methylD-aspartate and metabotropic glutamate receptors. Synapse 65, 795-803. doi: 10.1002/syn.20906

Hung, A. Y., Futai, K., Sala, C., Valtschanoff, J. G., Ryu, J., Woodworth, M. A., et al. (2008). Smaller dendritic spines, weaker synaptic transmission, but enhanced spatial learning in mice lacking Shank1. J. Neurosci. 28, 1697-1708. doi: 10.1523/JNEUROSCI.3032-07.2008

Kim, Y., Zhou, P., Qian, L., Chuang, J. Z., Lee, J., Li, C., et al. (2007). MyD88-5 links mitochondria, microtubules, and JNK3 in neurons and regulates neuronal survival. J. Exp. Med. 204, 2063-2074. doi: 10.1084/jem.20070868

Lee, S. J., Kim, H. E., Choi, S. E., Shin, H. C., Kwag, W. J., Lee, B. K., et al. (2009). Involvement of $\mathrm{Ca}^{2+} /$ calmodulin kinase II (CaMK II) in genistein-induced potentiation of leucine/glutamine-stimulated insulin secretion. Mol. Cells 28, 167-174. doi: 10.1007/s10059-009-0119-7

Li, X. M., Li, C. C., Yu, S. S., Chen, J. T., Sabapathy, K., and Ruan, D. Y. (2007). JNK1 contributes to metabotropic glutamate receptor-dependent long-term depression and short-term synaptic plasticity in the mice area hippocampal CA1. Eur. J. Neurosci. 25, 391-396. doi: 10.1111/j.1460-9568.2006.05300.x
Lin, C. W., and Hsueh, Y. P. (2014). Sarm1, a neuronal inflammatory regulator, controls social interaction, associative memory and cognitive flexibility in mice. Brain Behav. Immun. 37, 142-151. doi: 10.1016/j.bbi.2013.12.002

Lin, C. W., Liu, H. Y., Chen, C. Y., and Hsueh, Y. P. (2014). Neuronally-expressed Sarml regulates expression of inflammatory and antiviral cytokines in brains. Innate Immun. 20, 161-172. doi: 10.1177/1753425913485877

Lin, H. C., Gean, P. W., Wang, C. C., Chan, Y. H., and Chen, P. S. (2013). The amygdala excitatory/inhibitory balance in a valproate-induced rat autism model. PLoS ONE 8:e55248. doi: 10.1371/journal.pone.0055248

Lisman, J., Schulman, H., and Cline, H. (2002). The molecular basis of CaMKII function in synaptic and behavioural memory. Nat. Rev. Neurosci. 3, 175-190. doi: 10.1038/nrn753

Moult, P. R., Correa, S. A., Collingridge, G. L., Fitzjohn, S. M., and Bashir, Z. I. (2008). Co-activation of p38 mitogen-activated protein kinase and protein tyrosine phosphatase underlies metabotropic glutamate receptor-dependent long-term depression. J. Physiol. 586, 2499-2510. doi: 10.1113/jphysiol.2008.153122

Nuytens, K., Gantois, I., Stijnen, P., Iscru, E., Laeremans, A., Serneels, L., et al. (2013). Haploinsufficiency of the autism candidate gene Neurobeachin induces autism-like behaviors and affects cellular and molecular processes of synaptic plasticity in mice. Neurobiol. Dis. 51, 144-151. doi: 10.1016/j.nbd.2012.11.004

O’Roak, B. J., Vives, L., Fu, W., Egertson, J. D., Stanaway, I. B., Phelps, I. G., et al. (2012). Multiplex targeted sequencing identifies recurrently mutated genes in autism spectrum disorders. Science 338, 1619-1622. doi: 10.1126/science.1227764 Oh, W. C., Hill, T. C., and Zito, K. (2013). Synapse-specific and size-dependent mechanisms of spine structural plasticity accompanying synaptic weakening. Proc. Natl. Acad. Sci. U.S.A. 110, E305-E312. doi: 10.1073/pnas.1214705110

Pardo, C., Azhagiri, A., Lawler, C., and Zea-Vera, A. (2009). "Expression profiling of TLR signaling pathway genes in brain tissue from patients with autism," in International Meeting for Autism Research (West Hartford, CT: The International Society for Autism Research).

Peca, J., Feliciano, C., Ting, J. T., Wang, W., Wells, M. F., Venkatraman, T. N., et al. (2011). Shank3 mutant mice display autistic-like behaviours and striatal dysfunction. Nature 472, 437-442. doi: 10.1038/nature09965

Pinto, D., Pagnamenta, A. T., Klei, L., Anney, R., Merico, D., Regan, R., et al. (2010). Functional impact of global rare copy number variation in autism spectrum disorders. Nature 466, 368-372. doi: 10.1038/nature09146

Ramiro-Cortes, Y., and Israely, I. (2013). Long lasting protein synthesis- and activitydependent spine shrinkage and elimination after synaptic depression. PLoS ONE 8:e71155. doi: 10.1371/journal.pone.0071155

Sato, D., Lionel, A. C., Leblond, C. S., Prasad, A., Pinto, D., Walker, S., et al. (2012). SHANK1 deletions in males with autism spectrum disorder. Am. J. Hum. Genet. 90, 879-887. doi: 10.1016/j.ajhg.2012.03.017

Schmeisser, M. J., Ey, E., Wegener, S., Bockmann, J., Stempel, A. V., Kuebler, A., et al. (2012). Autistic-like behaviours and hyperactivity in mice lacking ProSAP1/Shank2. Nature 486, 256-260. doi: 10.1038/nature11015

Sui, L., and Chen, M. (2012). Prenatal exposure to valproic acid enhances synaptic plasticity in the medial prefrontal cortex and fear memories. Brain Res. Bull. 87, 556-563. doi: 10.1016/j.brainresbull.2012.01.011

Szretter, K. J., Samuel, M. A., Gilfillan, S., Fuchs, A., Colonna, M., and Diamond, M. S. (2009). The immune adaptor molecule SARM modulates tumor necrosis factor alpha production and microglia activation in the brainstem and restricts West Nile Virus pathogenesis. J. Virol. 83, 9329-9338. doi: 10.1128/JVI.00836-09 Verpelli, C., Dvoretskova, E., Vicidomini, C., Rossi, F., Chiappalone, M., Schoen, M., et al. (2011). Importance of Shank3 protein in regulating metabotropic glutamate receptor 5 (mGluR5) expression and signaling at synapses. J. Biol. Chem. 286, 34839-34850. doi: 10.1074/jbc.M111.258384

Wang, H. F., Shih, Y. T., Chen, C. Y., Chao, H. W., Lee, M. J., and Hsueh, Y. P. (2011). Valosin-containing protein and neurofibromin interact to regulate dendritic spine density. J. Clin. Invest. 121, 4820-4837. doi: 10.1172/JCI45677

Won, H., Lee, H. R., Gee, H. Y., Mah, W., Kim, J. I., Lee, J., et al. (2012). Autistic-like social behaviour in Shank2-mutant mice improved by restoring NMDA receptor function. Nature 486, 261-265. doi: 10.1038/nature11208

Zoghbi, H. Y., and Bear, M. F. (2012). Synaptic dysfunction in neurodevelopmental disorders associated with autism and intellectual disabilities. Cold Spring Harb. Perspect. Biol. 4:a009886. doi: 10.1101/cshperspect.a009886

Conflict of Interest Statement: The authors declare that the research was conducted in the absence of any commercial or financial relationships that could be construed as a potential conflict of interest. 
Received: 02 January 2014; accepted: 09 March 2014; published online: 01 April 2014. Citation: Lin C-W, Chen C-Y, Cheng S-J, Hu H-T and Hsueh Y-P (2014) Sarm1 deficiency impairs synaptic function and leads to behavioral deficits, which can be ameliorated by an mGluR allosteric modulator. Front. Cell. Neurosci. 8:87. doi: 10.3389/fncel.2014.00087

This article was submitted to the journal Frontiers in Cellular Neuroscience.
Copyright (c) 2014 Lin, Chen, Cheng, Hu and Hsueh. This is an open-access article distributed under the terms of the Creative Commons Attribution License (CC BY). The use, distribution or reproduction in other forums is permitted, provided the original author(s) or licensor are credited and that the original publication in this journal is cited, in accordance with accepted academic practice. No use, distribution or reproduction is permitted which does not comply with these terms. 